\title{
Nanoparticle-based Paclitaxel Suspension
}

National Cancer Institute

\section{Source}

National Cancer Institute. Nanoparticle-based Paclitaxel Suspension. NCI Thesaurus.

Code C118648.

A nanoparticle-based suspension containing the water-insoluble taxane paclitaxel, with potential antineoplastic activity. Upon intraperitoneal administration, paclitaxel binds to tubulin and inhibits the disassembly of microtubules, which leads to the inhibition of cell division. The nanoparticle-based formulation is devoid of toxic solvents, such as cremophor; therefore, this agent has fewer side effects than the standard, solventbased paclitaxel formulation. 